\title{
Impact of low hemoglobin on the development of contrast-induced nephropathy: A retrospective cohort study
}

\author{
JINZHONG XU ${ }^{1,2^{*}}$, MEILING ZHANG $^{3 *}$, YINGHUA NI $^{4 *}$, JIANA SHI $^{5}$, RANRAN GAO $^{6}$,

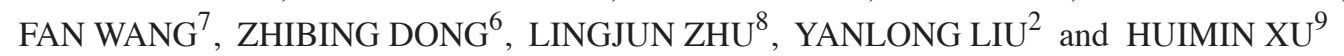 \\ ${ }^{1}$ Department of Clinical Pharmacy, The Affiliated Wenling Hospital of Wenzhou Medical University, Wenling, \\ Zhejiang 317500; ${ }^{2}$ College of Pharmaceutical Sciences, Wenzhou Medical University, Wenzhou, Zhejiang 325035; \\ ${ }^{3}$ Department of Pharmacy, Tongde Hospital of Zhejiang Province, Hangzhou, Zhejiang 310012; ${ }^{4}$ Department of \\ Clinical Pharmacy, The Children's Hospital, Zhejiang University School of Medicine, Hangzhou, Zhejiang 310052; \\ ${ }^{5}$ Department of Pharmacy, Zhejiang Province People's Hospital, Hangzhou, Zhejiang 31014; ${ }^{6}$ Department of \\ Cardiovascular Medicine, The Affiliated Wenling Hospital of Wenzhou Medical University, Wenling, Zhejiang 317500; \\ ${ }^{7}$ Beijing Hui-Long-Guan Hospital, Peking University, Beijing 100096; Departments of ${ }^{8}$ Cardiovascular Medicine \\ and ${ }^{9}$ Clinical Pharmacy, The Second Affiliated Hospital of Zhejiang University \\ School of Medicine, Hangzhou, Zhejiang 310009, P.R. China
}

Received May 7, 2015; Accepted March 31, 2016

DOI: $10.3892 / \mathrm{etm} .2016 .3416$

\begin{abstract}
An increase in the use of iodinated contrast media, such as iohexol, iodixanol, iopamidol and iopromide, occasionally causes contrast-induced nephropathy (CIN) in patients undergoing coronary angiography (CAG) and/or percutaneous coronary intervention (PCI). The present study aimed to assess the effects of low levels of hemoglobin on the development of CIN in patients with normal renal function following CAG/PCI. A total of 841 consecutive patients undergoing CAG/PCI were divided into two groups: Patients with low levels of hemoglobin (male, $<120 \mathrm{~g} / \mathrm{l}$; female, $<110 \mathrm{~g} / \mathrm{l} ; \mathrm{n}=156$ ) and normal levels of hemoglobin (male, 120-160 g/l; female, $110-150 \mathrm{~g} / \mathrm{l} ; \mathrm{n}=685$ ). Multiple logistic regression analysis was performed to identify risk factors for CIN, which developed in $14.7 \%$ of patients with low levels of hemoglobin (relative
\end{abstract}

Correspondence to: Dr Yanlong Liu, College of Pharmaceutical Sciences, Wenzhou Medical University, 268 Xueyuan West Road, Wenzhou, Zhejiang 325035, P.R. China

Email: benjaminlyl@wmu.edu.cn

Dr Huimin Xu, Department of Clinical Pharmacy, The Second Affiliated Hospital of Zhejiang University School of Medicine, 88 Jiefang Road, Hangzhou, Zhejiang 310009, P.R. China

E-mail: xuhuiminze@126.com

*Contributed equally

Abbreviations: CAG, coronary angiography; CIN, contrast-induced nephropathy; PCI, percutaneous coronary intervention

Key words: low hemoglobin, contrast media, contrast-induced nephropathy risk, 3.07) and 5\% of patients with normal levels of hemoglobin $(\mathrm{P}<0.01)$. Independent risk factors for developing CIN in patients with low levels of hemoglobin were a contrast media volume $\geq 200 \mathrm{ml}$, diuretic usage, low levels of hemoglobin and diabetes mellitus. For the patients with normal hemoglobin levels, the independent risk factors for developing CIN were a contrast media volume $\geq 200 \mathrm{ml}$ and diuretic usage. The change in serum creatinine in patients with low levels of hemoglobin was significantly greater compared with patients with normal levels of hemoglobin $(7.35 \pm 22.60$ vs. $1.40 \pm 12.00$; $\mathrm{P}<0.01)$. A similar incidence of developing CIN was observed when patients were administered each type of contrast media: Iohexol, iodixanol, iopamidol and iopromide. The optimal cut-off point at which the serum hemoglobin concentration resulted in a high probability of developing CIN was determined as $111.5 \mathrm{~g} / \mathrm{l}$ in females and $115.5 \mathrm{~g} / \mathrm{l}$ in males. In conclusion, low levels of hemoglobin were observed to be an independent risk factor for developing CIN. Patients with reduced hemoglobin levels should, therefore, be closely monitored prior to, and during, the administration of iodinated contrast media.

\section{Introduction}

Iodinated contrast media used in diagnostic and therapeutic procedures often causes nephrotoxicity (1). Contrast-induced nephropathy (CIN) is one of the main adverse effects of iodinated contrast media in patients undergoing coronary angiography (CAG) and/or percutaneous coronary intervention (PCI) (2). CIN refers to an impairment of renal function (usually defined as the elevation of serum creatinine levels by $0.5 \mathrm{mg} / \mathrm{dl}$ or $25 \%$ ) that occurs within 3 days following intravascular administration of contrast media, with the exclusion of other causes $(3,4)$. CIN is independently associated with a longer stay in hospital, and results in increased long-term 
mortality and increased costs of medical care that partly result from the prolonged hospital stay $(5,6)$.

The incidence of CIN can be as high as $50 \%$ for high risk patients $(3,4,7)$. Risk factors associated with CIN include the type and dosage of contrast media, concomitant nephrotoxic medication, inflammation, diabetes mellitus, renal insufficiency, congestive heart failure, increasing age and female gender (5-10). The presence of multiple risk factors concomitantly can increase the risk of CIN disproportionately (7). A key step in minimizing the risk of developing CIN is to identify patients at risk and initiate appropriate prophylactic measures (6).

When multiple risk factors coexist, patients with low baseline hematocrit are reported to have a higher incidence of CIN (11), suggesting that anemia may be a risk factor for CIN. Low levels of hemoglobin may cause hypoxia owing to the decreased oxygen transport capacity (12) and their increased affinity for oxygen when exposed to contrast media (13), which aggravates hypoxic injury and further results in renal dysfunction $(14,15)$. However, few studies have assessed the effects of low hemoglobin levels on CIN in patients without baseline renal insufficiency. In the present study, an investigation into whether low levels of hemoglobin are associated with an increased incidence of CIN in patients undergoing CAG and/or PCI was performed.

\section{Materials and methods}

Study population and exclusion criteria. The present retrospective study included 841 patients (565 males and 276 females; aged $64.92 \pm 10.80$ years) who had been diagnosed with coronary heart disease and had undergone CAG/PCI at the Second Affiliated Hospital of Zhejiang University School of Medicine (Hangzhou, China), the Affiliated Wenling Hospital of Wenzhou Medical University (Wenling, China) or Tongde Hospital of Zhejiang Province (Hangzhou, China) between January 2013 and December 2013. Patients with baseline renal insufficiency, tumor-associated anemia, severe infection, cardiogenic shock and any diseases resulting in abnormal levels of hemoglobin (such as chronic pulmonary heart disease and congestive heart failure) were excluded from the present study. Patients who had been exposed to nephrotoxic medication (aminoglycosides and non-steroidal anti-inflammatory drugs, with the exception of aspirin), $\mathrm{N}$-acetylcysteine and interventional anemia management, with the exception of patients who had a diet of enriched foods prior to developing CIN, were also excluded. For patients who underwent multiple CAG/PCI procedures during the study period, only the first procedure was included for analysis.

In the study population, patients with comorbid coronary heart diseases, hypertension and diabetes mellitus, medication or treatment was provided according to corresponding guidelines, including the following: i) The European Society of Cardiology Guidelines for the management of acute myocardial infarction in patients presenting with ST-segment elevation or without persistent ST-segment elevation; ii) the guidelines of Chinese Hypertension League for the management of hypertension; and iii) Chinese Diabetes Society Guidelines for the management of diabetes (16-19). The demographic, clinical and laboratory data, including details of CAG/PCI, were collected from study patients. Written informed consent was obtained from all patients.

Clinical definitions. Patients were divided into the following two groups according to their hemoglobin levels: i) Patients with low hemoglobin levels (hemoglobin-L group; $n=156$ ); ii) and patients with normal hemoglobin levels (hemoglobin-N group; $n=685$ ). Baseline renal insufficiency was defined as an increase in serum creatinine concentration $>133 \mu \mathrm{mol} / 1$ (20). CIN was defined as an acute deterioration in renal function with an increase in serum creatinine of $0.5 \mathrm{mg} / \mathrm{dl}(50 \mu \mathrm{mol} / \mathrm{l})$, or $25 \%$ from the baseline within $96 \mathrm{~h}$ following the intravascular administration of contrast agents, in the absence of alternative risk factors. Anemia was defined as a hemoglobin concentration $<120 \mathrm{~g} / 1$ for males and $<110 \mathrm{~g} / \mathrm{l}$ for females, in accordance with the Chinese criteria (21).

Treatment and measurements. The patients were exposed to four contrast media, as follows: Iohexol [100 ml (30 g/l) or $50 \mathrm{ml}$ (15 g/l); Shanghai General Pharmaceutical, Co., Ltd., Shanghai, China]; iodixanol [100 ml (32 g/l) or $50 \mathrm{ml}(16 \mathrm{~g} / \mathrm{l})$; Shanghai General Pharmaceutical, Co., Ltd.]; iopamidol [100 ml (37 g/l) or $50 \mathrm{ml}$ (18.5 g/l); Sine Pharmaceutical, Co., Ltd., Shanghai, China]; and iopromide [100 ml (30 g/l) or $50 \mathrm{ml}$ (15 g/l); Bayer Vital GmbH, Leverkusen, Germany]. The patients received saline hydration within 3-12 $\mathrm{h}$ prior to and 6-24 h following CAG/PCI procedures, as described previously (22). Patient demographic, clinical and laboratory data, including details of the CAG/PCI, were collected. For patients with diabetes mellitus, the administration of metformin was withheld at least $24 \mathrm{~h}$ prior to the procedure, and replaced with insulin (NovoRapid 30, Novolin 30R or Novolin 70/30; Novo Nordisk A/S, Bagsvaerd, Denmark) or oral anti-diabetic drugs, including repaglinide tablets (Hansoh Pharmaceutical Co., Ltd., Jiangsu, China), Nateglinide tablets (Beijing Novartis Pharma Ltd., Beijing, China) or Acarbose tablets (Bayer HealthCare Company Ltd., Beijing, China). Blood samples $(5 \mathrm{ml})$ were measured for serum creatinine, glucose, uric acid and lipids using an automatic biochemical analyzer (Roche Module P800; Roche Diagnostics, Basel, Switzerland) and hemoglobin was detected using a hematology analyzer (XE-2100; Sysmex Corporation, Kobe, Japan).

The baseline characteristics of patients in the hemoglobin-L and hemoglobin-N groups were compared. The incidence of $\mathrm{CIN}$ and changes in serum creatinine prior to and following CAG/PCI were analyzed. Serum creatinine measurements taken prior to $\mathrm{CAG} / \mathrm{PCI}$ were recorded as the baseline creatinine level, and the post-procedure serum creatinine was recorded as the maximum creatinine level that was measured within 96 hours of CAG/PCI.

Statistical analysis. Statistical analysis was performed using SPSS version 20.0 (IBM SPSS, Armonk, NY, USA). Continuous data were expressed as the mean \pm standard deviation when normally distributed. Categorical data were expressed as the absolute value and percentage. Comparisons of continuous variables among the four contrast media were performed using one-way analysis of variance with multiple Scheffe-type comparisons after reciprocal transformation of the variables to correct for heterogeneity of variance. In order 
Table I. Baseline characteristics of patients with normal (Hemoglobin-N) or low (Hemoglobin-L) levels of hemoglobin.

\begin{tabular}{|c|c|c|c|}
\hline Parameter & Hemoglobin-N group $(n=685)$ & Hemoglobin-L group $(n=156)$ & P-value \\
\hline Female, n (\%) & $218(31.8 \%)$ & $58(37.2 \%)$ & 0.20 \\
\hline Age (years) & $63.56 \pm 10.72$ & $70.79 \pm 9.00$ & $<0.01$ \\
\hline Body weight (kg) & $63.17 \pm 11.44$ & $61.95 \pm 12.05$ & 0.24 \\
\hline Hypertension, n (\%) & $421(61.5 \%)$ & $100(64.1 \%)$ & 0.54 \\
\hline Diabetes mellitus, n (\%) & $135(19.7 \%)$ & $39(25.0 \%)$ & 0.14 \\
\hline Current smoking, n (\%) & $172(25.1 \%)$ & $30(19.2 \%)$ & 0.12 \\
\hline \multicolumn{4}{|l|}{ Medication used } \\
\hline Aspirin, n (\%) & $672(98.1 \%)$ & $153(98.1 \%)$ & 1.00 \\
\hline Clopidogrel, n (\%) & $667(97.4 \%)$ & $150(96.2 \%)$ & 0.42 \\
\hline ACEI/ARB, n (\%) & $519(75.8 \%)$ & $127(81.4 \%)$ & 0.13 \\
\hline$\beta$-blocker, n (\%) & $468(68.3 \%)$ & $109(69.9 \%)$ & 0.70 \\
\hline Statin, n $(\%)$ & $653(95.3 \%)$ & $151(96.8 \%)$ & 0.42 \\
\hline Anticoagulants, n (\%) & $300(43.8 \%)$ & $75(48.1 \%)$ & 0.33 \\
\hline $\mathrm{CCB}, \mathrm{n}(\%)$ & $192(28.0 \%)$ & $23(14.7 \%)$ & $<0.01$ \\
\hline PPI, n (\%) & $339(49.5 \%)$ & $102(65.4 \%)$ & $<0.01$ \\
\hline Nitrate, n (\%) & $305(44.5 \%)$ & $74(46.8 \%)$ & 0.60 \\
\hline Diuretics, n (\%) & $139(20.3 \%)$ & $42(26.9 \%)$ & 0.069 \\
\hline $\operatorname{LVEF}(\%)$ & $60.98 \pm 9.63$ & $61.40 \pm 11.17$ & 0.67 \\
\hline Total cholesterol (mmol/l) & $4.41 \pm 1.14$ & $4.15 \pm 1.11$ & $<0.01$ \\
\hline Triglyceride (mmol/l) & $1.75 \pm 1.22$ & $1.30 \pm 0.74$ & $<0.01$ \\
\hline LDL-C (mmol/l) & $2.64 \pm 0.97$ & $2.51 \pm 0.95$ & 0.13 \\
\hline Fasting blood glucose (mmol/l) & $5.88 \pm 1.96$ & $5.85 \pm 1.87$ & 0.90 \\
\hline Uric acid $(\mu \mathrm{mol} / \mathrm{l})$ & $355.85 \pm 98.12$ & $356.20 \pm 120.69$ & 0.97 \\
\hline Red blood cell $\left(10^{12} / 1\right)$ & $4.39 \pm 0.43$ & $3.61 \pm 0.44$ & $<0.01$ \\
\hline Hemoglobin $(\mathrm{g} / \mathrm{l})$ & $134.04 \pm 12.70$ & $105.11 \pm 11.39$ & $<0.01$ \\
\hline Hematocrit $(\%)$ & $0.40 \pm 0.039$ & $0.33 \pm 0.057$ & $<0.01$ \\
\hline
\end{tabular}

Data are expressed as the mean + standard deviation or number $(\%)$ of patients. ACEI, acetylcholinesterase inhibitor; ARB, angiotensin receptor blocker; CCB, calcium channel blocker; PPI, proton pump inhibitor; LVEF, left ventricular ejection fraction; LDL-C, low density lipoprotein cholesterol.

to evaluate inter- and intra-group differences, continuous variables were compared using the paired t-test and independent-samples t-test, and categorical variables were compared using a $\chi^{2}$ test or Fisher's exact test where appropriate. The Bonferroni correction was used for multiple comparisons. Univariate logistic regression was performed to search for the potential factor of CIN as a dependent variable. Variables that were statistically significant on univariate analysis and other potential variables were identified as predictors of CIN in the final multivariate model using the forward selection method. A two-sided $95 \%$ confidence interval (CI) was constructed around the point estimate of the relative risk. Receiver operating characteristic (ROC) curve analysis was performed to predict CIN. All tests were two-sided, and $\mathrm{P}<0.05$ was considered to indicate a statistically significant difference.

\section{Results}

Patient characteristics. As presented in Table I, patients in the hemoglobin-L group were significantly older compared with patients in the hemoglobin-N group $(70.79 \pm 9.00$ vs. 63 .
$56 \pm 10.72$ years; $\mathrm{P}<0.01)$. The plasma levels of total cholesterol, triglycerides, red blood cells, hemoglobin and hematocrit in the hemoglobin-L group were significantly lower compared with those in the hemoglobin-N group $(\mathrm{P}<0.05)$. In the hemoglobin- $\mathrm{L}$ group, a greater number of patients used calcium channel blockers and a lower number of patients used proton pump inhibitors compared with the hemoglobin-N group $(\mathrm{P}<0.05)$. There was no significant difference between the groups regarding the incidence of CIN resulting from the contrast media used $(\mathrm{P}=0.39$; Table II). Patients in both groups had been administered similar volumes of iohexol, iodixanol or iopamidol ( $\mathrm{P}>0.05)$, but only a small volume of iopromide $(\mathrm{P}<0.01)$.

Changes in levels of serum creatinine, incidence of CIN and hospital stay. The mean serum creatinine concentration levels increased significantly following the intravascular administration of contrast media in both patient groups $(\mathrm{P}<0.05$; Table III). However, the change in serum creatinine concentration levels prior to and following the use of contrast media in the hemoglobin-L group was significantly higher compared with the hemoglobin-N group $(\mathrm{P}<0.01)$. 
Table II. Contrast media volume used in patients undergoing CAG and/or PCI.

\begin{tabular}{|c|c|c|c|c|c|}
\hline Parameter & Iohexol (n=177) & Iodixanol $(\mathrm{n}=133)$ & Iopamidol $(\mathrm{n}=260)$ & Iopromide $(n=271)$ & P-value \\
\hline Mean volume (ml) & $137.49 \pm 86.40$ & $127.33 \pm 63.02$ & $125.63 \pm 64.30$ & $98.49 \pm 51.17^{\mathrm{a}}$ & $<0.01$ \\
\hline CIN, n (\%) & $10(5.6 \%)$ & $13(9.8 \%)$ & $19(7.3 \%)$ & $15(5.5 \%)$ & 0.39 \\
\hline
\end{tabular}

a $\mathrm{P}<0.01$, iopromide vs. iohexol, iodixanol or iopamidol; $\mathrm{P}=0.97$, iohexolvs.iodixanol; $\mathrm{P}=0.99$, iodixanol vs. iopamidol; $\mathrm{P}=0.85$, iohexol vs. iopamidol. CAG, coronary angiography; PCI, percutaneous intervention; CIN, contrast-induced nephropathy.

Table III. Changes in serum creatinine and incidence of contrast-induced nephropathy in patients with normal (Hemoglobin-N) and low (Hemoglobin-L) levels of hemoglobin.

\begin{tabular}{lccc}
\hline Parameter & Hemoglobin-N $(\mathrm{n}=685)$ & Hemoglobin-L $(\mathrm{n}=156)$ & P-value \\
\hline Serum creatinine before use of contrast media $(\mu \mathrm{mol} / \mathrm{l})$ & $78.00 \pm 18.41$ & $80.31 \pm 23.35$ & 0.31 \\
Serum creatinine after use of contrast media $(\mu \mathrm{mol} / \mathrm{l})$ & $79.40 \pm 18.67$ & $87.65 \pm 33.49$ & $<0.01$ \\
Absolute change in serum creatinine $(\mu \mathrm{mol} / \mathrm{l})$ & $1.40 \pm 12.00$ & $7.35 \pm 22.60$ & $<0.01$ \\
CIN & $34(5.0)$ & $23(14.7)$ & $<0.01$ \\
Females with CIN & $9(26.5)$ & $10(43.5)$ & 0.18 \\
Males with CIN & $25(73.5)$ & $13(56.5)$ & $<0.01$ \\
Total duration of hospital stay (days) & $7.52 \pm 4.03$ & $8.87 \pm 4.87$ & 0.15 \\
Duration of hospital stay after use of contrast media (days) & $4.59 \pm 3.55$ & $5.04 \pm 3.54$ & \\
\hline
\end{tabular}

Data are expressed as the mean \pm standard deviation or $\mathrm{n}(\%)$. CIN , contrast-induced nephropathy.

CIN occurred in $6.78 \%$ (57 of 841 patients) of the total patients in the study. Notably, there was no significant difference in the occurrence of CIN in genders between the two groups (females, 26.5 vs. $43.5 \%$; males, 73.5 vs. $56.5 \%$; $\mathrm{P}=0.18$; Table III). Among the 57 patients who developed CIN, $12(12 / 319,3.76 \%)$ had received $<100 \mathrm{ml}$ contrast media, 23 $(23 / 383,6.00 \%)$ received $100-200 \mathrm{ml}$ contrast media, and 22 $(22 / 139,15.83 \%)$ received $\geq 200 \mathrm{ml}$ contrast media (Fig. 1).

The mean total duration of hospital stay of patients in the hemoglobin-L group was $8.87 \pm 4.87$ days, which was significantly longer than that of patients in the hemoglobin-N group $(7.52 \pm 4.03$ days; $\mathrm{P}<0.01)$. The mean duration of hospital stay following administration of the contrast media was not significantly different between the two groups $(4.59 \pm 3.55$ vs. 5.04 \pm 3.54 days; $\mathrm{P}=0.15$ ).

Risk factors associated with CIN. In a univariate model, contrast media volume $\geq 200 \mathrm{ml}$, diuretic use, low levels of hemoglobin, diabetes mellitus, hyperuricemia, use of anticoagulants and age $\geq 70$ years were associated with the development of CIN $(\mathrm{P}<0.05)$. Multivariate analysis was performed to evaluate these baseline univariate predictors. Contrast media volume $\geq 200 \mathrm{ml}$ (RR, 4.64; 95\% CI, 2.15-9.99; $\mathrm{P}<0.01$ ), diuretic usage (RR, 3.68; 95\% CI, 2.07-6.53; $\mathrm{P}<0.01$ ), low hemoglobin levels (RR, 3.07; 95\% CI, 1.69-5.56; $\mathrm{P}<0.01$ ) and diabetes mellitus (RR, 2.46; 95\% CI, 1.35-4.46; $\mathrm{P}<0.01$ ) were found to be associated with an increased risk of CIN in the hemoglobin-L group (Table IV). For the patients with normal hemoglobin levels, contrast media volume $\geq 200 \mathrm{ml}$ (RR, 4.56; 95\% CI, 1.73-12.03; $\mathrm{P}<0.01)$ and diuretic usage (RR,

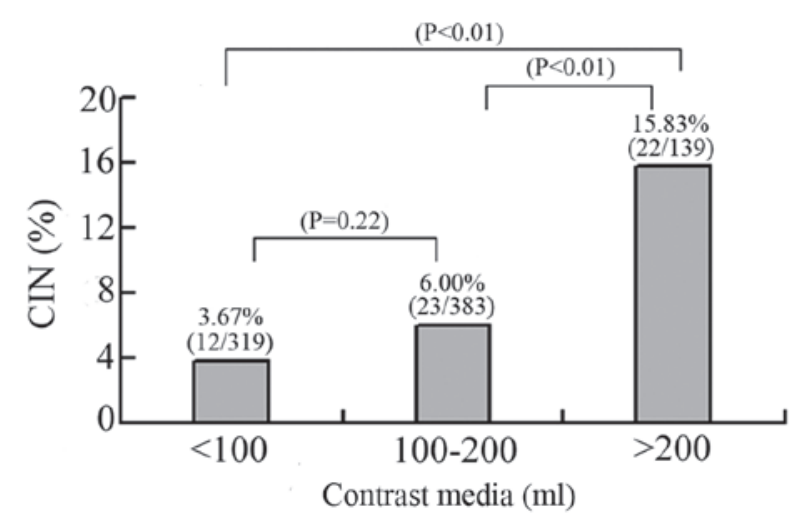

Figure 1. Incidence of CIN in patients who received different volumes of contrast media. A $\chi^{2}$ test was performed to detect inter-group comparison. CIN, contrast-induced nephropathy.

3.31; 95\% CI, 1.62-6.76; $\mathrm{P}<0.01)$ were shown to be associated with an increased risk of CIN (Table V).

ROC. ROC curve analysis was conducted to determine the cut-off point at which there is a high probability of developing CIN. Male and female patients were analyzed separately. It was observed that the optimal cut-off point of serum hemoglobin concentration for predicting CIN was $111.5 \mathrm{~g} / 1$ in females, with a sensitivity of $63.2 \%$ and specificity of $76.3 \%$, and an area under the curve (AUC) of $0.737(\mathrm{P}<0.01$; Fig. 2). A serum hemoglobin concentration of $115.5 \mathrm{~g} / \mathrm{l}$ was determined as the optimal cut-off point for predicting CIN in males, with a sensitivity of $34.2 \%$ and specificity of $89.2 \%$, and an AUC of 0.625 
Table IV. Risk factors associated with contrast-induced nephropathy in the hemoglobin-L group were determined by univariate and multivariate logistic regression analyses.

\begin{tabular}{|c|c|c|c|c|c|c|}
\hline \multirow[b]{2}{*}{ Variable } & \multicolumn{3}{|c|}{ Univariate analysis } & \multicolumn{3}{|c|}{ Multivariate analysis } \\
\hline & $\mathrm{RR}$ & $95 \% \mathrm{CI}$ & P-value & $\mathrm{RR}$ & $95 \% \mathrm{CI}$ & P-value \\
\hline Contrast media volume $\geq 200 \mathrm{ml}$ & 4.81 & $2.31-10.03$ & $<0.01$ & 4.64 & $2.15-9.99$ & $<0.01$ \\
\hline Use of diuretics & 3.92 & $2.27-6.78$ & $<0.01$ & 3.68 & $2.07-6.53$ & $<0.01$ \\
\hline Low levels of hemoglobin & 3.31 & $1.89-5.80$ & $<0.01$ & 3.07 & $1.69-5.56$ & $<0.01$ \\
\hline Diabetes mellitus & 2.61 & $1.49-4.58$ & $<0.01$ & 2.46 & $1.35-4.46$ & $<0.01$ \\
\hline Hypercholesterolemia & 2.14 & $1.23-3.70$ & $<0.01$ & & & \\
\hline Use of anticoagulants & 2.44 & $1.39-4.28$ & $<0.05$ & & & \\
\hline Age $\geq 70$ years & 1.83 & $1.07-3.14$ & $<0.05$ & & & \\
\hline Use of PPI & 1.60 & $0.92-2.80$ & 0.096 & & & \\
\hline Contrast media volume $100-200 \mathrm{ml}$ & 1.63 & $0.80-3.34$ & 0.18 & & & \\
\hline LDL-C & 1.32 & $0.70-2.52$ & 0.39 & & & \\
\hline Use of nitrates & 1.20 & $0.70-2.05$ & 0.51 & & & \\
\hline Use of statins & 1.29 & $0.30-5.48$ & 0.74 & & & \\
\hline Gender (female) & 1.025 & $0.58-1.81$ & 0.93 & & & \\
\hline Use of ACEI & 1.02 & $0.54-1.94$ & 0.94 & & & \\
\hline
\end{tabular}

RR, relative risk; CI, confidence interval; PPI, proton pump inhibitor; LDL-C, low-density lipoprotein cholesterol; ACEI, acetylcholinesterase inhibitor.

Table V. Risk factors associated with contrast-induced nephropathy in the hemoglobin-N group were determined by univariate and multivariate logistic regression analyses.

\begin{tabular}{|c|c|c|c|c|c|c|}
\hline \multirow[b]{2}{*}{ Variable } & \multicolumn{3}{|c|}{ Univariate analysis } & \multicolumn{3}{|c|}{ Multivariate analysis } \\
\hline & RR & $95 \% \mathrm{CI}$ & P-value & RR & $95 \% \mathrm{CI}$ & P-value \\
\hline Contrast media volume $\geq 200 \mathrm{ml}$ & 4.53 & $1.73-11.82$ & $<0.01$ & 4.56 & $1.73-12.03$ & $<0.01$ \\
\hline Use of diuretics & 3.29 & $1.63-6.65$ & $<0.01$ & 3.31 & $1.62-6.76$ & $<0.01$ \\
\hline Diabetes mellitus & 2.34 & $1.13-4.86$ & 0.02 & & & \\
\hline Use of anticoagulants & 1.30 & $0.65-2.59$ & 0.46 & & & \\
\hline Age & 1.30 & $0.63-2.69$ & 0.47 & & & \\
\hline Use of PPI & 1.69 & $0.83-3.44$ & 0.15 & & & \\
\hline Contrast media volume $100-200 \mathrm{ml}$ & 1.97 & $0.79-4.91$ & 0.15 & & & \\
\hline LDL-C & 1.33 & $0.59-3.02$ & 0.49 & & & \\
\hline Use of nitrates & 1.43 & $0.72-2.85$ & 0.31 & & & \\
\hline Use of ACEI & 1.25 & $0.53-2.92$ & 0.61 & & & \\
\hline Smoking & 1.08 & $0.49-2.36$ & 0.85 & & & \\
\hline Total cholesterol & 1.05 & $0.46-2.36$ & 0.91 & & & \\
\hline
\end{tabular}

RR, relative risk; CI, confidence interval; PPI, proton pump inhibitor; LDL-C, low-density lipoprotein cholesterol; ACEI, acetylcholinesterase inhibitor.

( $\mathrm{P}<0.01$; Fig. 3). Maintaining a concentration of hemoglobin $>130 \mathrm{~g} / 1$ decreased the incidence of CIN by $26.3 \%$ and $25.8 \%$ for males and females respectively.

\section{Discussion}

The present study demonstrated that patients with low levels of hemoglobin have a nearly three-fold higher incidence of developing CIN compared with patients with normal levels of hemoglobin, suggesting that low hemoglobin is a strong risk factor for developing CIN. Furthermore, patients with low hemoglobin levels had longer total hospital stay. The cut-off hemoglobin values for predicting CIN were $115.5 \mathrm{~g} / 1$ for male and $111.5 \mathrm{~g} / \mathrm{l}$ for female patients.

In the current study, although the patients with low levels of hemoglobin were older, multivariate analysis did not identify 


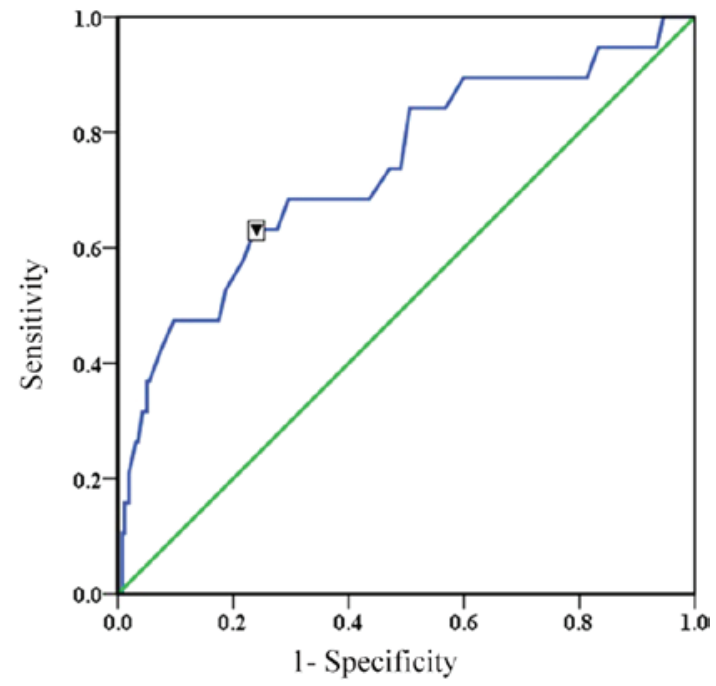

Figure 2. Receiver operating characteristic (ROC) curve analysis of serum hemoglobin concentration for predicting contrast-induced nephropathy in female patients. The triangle marks the cut-off point $(111.5 \mathrm{~g} / \mathrm{l})$ for developing CIN; sensitivity, 63.2\%; specificity, $76.3 \%$; area under the ROC curve, 0.737; $95 \%$ CI, $0.607-0.866 ; \mathrm{P}<0.01$.

age $\geq 70$ years as a risk factor. It has been reported that patients $\geq 70$ years have an increased risk of CIN $(23,24)$; however, the reasoning behind this is multifactorial and partially due to the reduced renal function typically observed in elderly patients (7). Thus, it is reasonable to account for advanced age when planning the administration of contrast media.

Four different contrast media were investigated in the present study, including the iso-osmolality medium iodixanol and the low-osmolality media iohexol, iopamidol and iopromide. Iso-osmolality media are believed to cause a low incidence of CIN in comparison with low-osmolality media; however, this remains controversial (23). Solomon (25) reported that the risk of CIN was significantly lower with iodixanol and iopamidol compared with iohexol, but no significant difference was observed between iodixanol and iopamidol. In an earlier meta-analysis, it was observed that there was no difference in the incidence of CIN in patients without pre-existing renal insufficiency, or injury based on the use of contrast media with different osmolalities (26), and these results were consistent with the findings of the present study.

Besides renal injury and changes in serum creatinine, other risk factors of CIN have been reported, including high-dose contrast media, diuretic usage and diabetes (26-29). A previous study reported that the incidence of CIN increases with higher doses of contrast media (27), and in the present study, patients receiving $\geq 200 \mathrm{ml}$ contrast media were more likely to suffer from CIN. However, patients had a similar risk of developing CIN when receiving $<100$ or $100-200 \mathrm{ml}$ contrast media, suggesting that a potential safe range of contrast media is $<200 \mathrm{ml}$. Thus, it is possible to decrease the incidence of CIN in patients undergoing CAG/PCI by reducing the dose of contrast media to $<200 \mathrm{ml}$.

The association of diuretics with the development of CIN may be a result of their nephrotoxic potential, particularly in patients with pre-existing renal diseases (28). Patients treated with diuretics have been reported to have a greater incidence

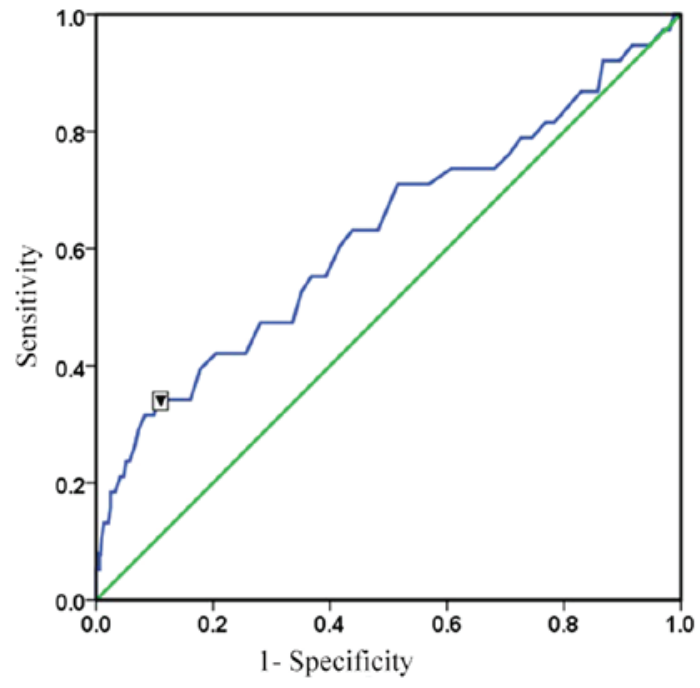

Figure 3. Receiver operating characteristic (ROC) curve analysis of serum hemoglobin concentration for predicting contrast-induced nephropathy in male patients. The triangle marks the cut-off point $(115.5 \mathrm{~g} / \mathrm{l})$ for developing CIN; sensitivity, $34.2 \%$; specificity, $89.2 \%$; area under the ROC curve, 0.625 ; $95 \%$ CI, 0.520-0.729; $\mathrm{P}=0.01$

of CIN (30), and it is recommended that diuretics should be discontinued for at least $24 \mathrm{~h}$ prior to the administration of contrast media, particularly when the glomerular filtration rate is $<60 \mathrm{ml} / \mathrm{min}$ per $1.73 \mathrm{~m}^{2}$ (28). The present study determined that the use of diuretics resulted in a high risk of developing of CIN (RR, 3.68; 95\% CI, 2.07-6.53; P<0.01). However, diuretics have previously been reported to have a potentially protective effect against kidney injury $(31,32)$. Due to these conflicts of evidence, a meta-analysis was performed to examine the clinical efficacy of furosemide administration in preventing CIN (33). Unexpectedly, furosemide, as a diuretic, has no additional influence beyond saline hydration on the incidence of CIN (33). However, the impact of furosemide dose on clinical outcome was not taken into account. In the present study, the mean daily dose of furosemide in patients with CIN was $30.37 \pm 10.18 \mathrm{mg}$, which is a higher dose than that used in similar studies (31). Thus, it remains to be demonstrated whether a high dose of diuretics is related to a higher risk of developing CIN.

Diabetic nephropathy has been identified as a strong and independent risk factor for CIN (34), which is consistent with the findings of the present study. In addition, individuals with diabetes, even in the absence of nephropathy, are more likely to develop CIN compared with non-diabetic individuals (4). Therefore, diabetes mellitus as a risk factor for developing CIN should not be underestimated, and patients with diabetes mellitus, regardless of whether they have renal dysfunction, should be evaluated closely prior to the administration of contrast media.

The impact that low hemoglobin levels have on the development of CIN has not been clearly identified. Previous studies have concluded that low levels of hemoglobin are not a risk factor for CIN $(8,35,36)$, while one study observed that a reduction in hematocrit increased the incidence of CIN in patients with or without chronic kidney disease (RR, 1.23; 95\% CI, 1.14-1.31; P<0.01) (11). It is likely that a number of these studies did not rigorously exclude the confounders 
related to low hemoglobin or serum creatinine, such as chronic kidney disease. Low levels of hemoglobin may occur as a result of various conditions, such as immunological disease, malignancy, chemical exposure or chronic kidney disease, which results in difficulty in assessing the independent effects of hemoglobin on CIN (29,37-40). In the present study, however, low levels of hemoglobin were demonstrated to be an independent risk factor for CIN, increasing the risk by 3.07 (95\% CI, 1.69-5.56; $\mathrm{P}<0.01)$, although it was not the highest risk factor for CIN and was second to contrast media volume $\geq 200 \mathrm{ml}$ and the use of diuretics. An ROC analysis for male and female patients separately was performed in the present study, and it was determined that the different cut-off values of serum hemoglobin for developing CIN in males and females was different, emphasizing that gender should be taken into account when assessing the risk of developing CIN.

It is important to note that low hemoglobin levels are often associated with poor prognosis and mortality in patients (41). It has been reported that there is a dose-dependent increase in mortality when hemoglobin levels decrease below the optimal hemoglobin range (42). The risk of mortality increases 2.5 times for every gram reduction in hemoglobin $<80 \mathrm{mg} / \mathrm{l}$ (43). Furthermore, Shah et al (42) reported that the risk associated with low levels of hemoglobin is greater in patients with myocardial infarction than for those with stable angina. Therefore, a previous study treated anemic patients with myocardial injury with blood transfusions and demonstrated favorable outcomes (44). In addition, patients with coronary artery disease are given treatment to maintain their hemoglobin concentrations at a minimum of $100 \mathrm{~g} / 1$ (45). In each case, prophylactic blood transfusions may decrease the risk of developing CIN and the risk of mortality, in particular in anemic patients at risk of myocardial infarction.

In the present study, no severe clinical manifestations in the patients with CIN were detected, such as acute renal failure requiring dialysis or mortality resulted from CIN. In general, levels of serum creatinine typically peaked at 3-5 days following exposure to contrast agents, and returned to the baseline, or near baseline, level within 1-3 weeks following adequate hydration (46).

Several limitations of the present study should be noted, firstly that it is a retrospective study. Secondly, the renal function of patients was only assessed based on the increase in serum creatinine; no other indicators, such as glomerular filtration rate, were used. Thirdly, the present study included patients with multi-vessel and single coronary artery diseases, and the former may necessitate the use of higher volumes of contrast media. Finally, the hemoglobin level in populations is known to vary with altitude (47). The current study was performed in Southeast China, a region of low altitude. Thus, the results of the present study should be reviewed with caution.

In conclusion, patients with low levels of hemoglobin, including those with normal renal function, are at a higher risk of developing CIN. Therefore, the level of hemoglobin should be closely monitored in patients with low hemoglobin prior to administration of contrast media, particularly in those with hemoglobin levels below the cut-off point and at risk of developing CIN.

\section{Acknowledgements}

The present study was supported by grants from the Wenling Foundation of Science and Technology (no. 2011WLCB0109 and 2014C311051), the Natural Science Foundation of China (no. 81100993 and 81300311), the Zhejiang Natural Science Foundation (no. LY12H03001 and LQ13H280002) and the Research Development Fund of Wenzhou Medical University (no. QTJ15001).

\section{References}

1. Parfrey P, Griffiths S, Barrett B, Paul MD, Genge M, Withers J, Farid N and McManamon PJ: Contrast material-induced renal failure in patients with diabetes mellitus, renal insufficiency or both: A prospective controlled study. N Engl J Med 320: 143-149, 1989.

2. Gandhi S, Mosleh W, Abdel-Qadir H and Farkouh ME: Statins and contrast-induced acute kidney injury with coronary angiography. Am J Med 127: 987-1000, 2014.

3. Chertow GM, Christiansen CL, Cleary PD, Munro C and Lazarus JM: Prognostic stratification in critically ill patients with acute renal failure requiring dialysis. Arch Intern Med 155: 1505-1511, 1995.

4. Rihal CS, Textor SC, Grill DE, Berger PB, Ting HH, Best PJ, Singh M, Bell MR, Barsness GW, Mathew V, et al: Incidence and prognostic importance of acute renal failure after percutaneous coronary intervention. Circulation 105: 2259-2264, 2002.

5. Palli E, Makris D, Papanikolaou J, Garoufalis G and Zakynthinos E: Contrast-induced nephropathy in aged critically ill patients. Oxid Med Cell Longev 2014: 756469, 2014.

6. Kwasa EA, Vinayak S and Armstrong R: The role of inflammation in contrast-induced nephropathy. Br J Radiol 87: 20130738, 2014.

7. Pucelikova T, Dangas G and Mehran R: Contrast-induced nephropathy. Catheter Cardiovasc Interv 71: 62-72, 2008.

8. Toprak O, Cirit M, Yesil M, Bayata S, Tanrisev M, Varol U, Ersoy R and Esi E: Impact of diabetic and pre-diabetic state on development of contrast-induced nephropathy in patients with chronic kidney disease. Nephrol Dial Transplant 22: 819-826, 2007.

9. Mehran R and Nikolsky E: Contrast-induced nephropathy: Definition, epidemiology, and patients at risk. Kidney Int Suppl 69: S11-S15, 2006.

10. Lee J, Cho JY, Lee HJ, Jeong YY, Kim CK, Park BK, Sung DJ, Kang BC, Jung SI, Lee EJ, et al; Korean Society of Urogenital Radiology (KSUR); Korean Society of Radiology: Contrast-induced nephropathy in patients undergoing intravenous contrast-enhanced computed tomography in Korea: A multi-institutional study in 101487 patients. Korean J Radiol 15: 456-463, 2014.

11. Nikolsky E, Mehran R, Lasic Z, Mintz GS, Lansky AJ, Na Y, Pocock S, Negoita M, Moussa I, Stone GW, et al: Low hematocrit predicts contrast-induced nephropathy after percutaneous coronary interventions. Kidney Int 67: 706-713, 2005.

12. Celsing F, Svedenhag J, Pihlstedt P and Ekblom B: Effects of anaemia and stepwise-induced polycythaemia on maximal aerobic power in individuals with high and low haemoglobin concentrations. Acta Physiol Scand 129: 47-54, 1987.

13. Kim SJ, Salem MR, Joseph NJ, Madayag MA, Cavallino RP and Crystal GJ: Contrast media adversely affect oxyhemoglobin dissociation. Anesth Analg 71: 73-76, 1990.

14. Heyman SN, Evans RG, Rosen S and Rosenberger C: Cellular adaptive changes in AKI: Mitigating renal hypoxic injury. Nephrol Dial Transplant 27: 1721-1728, 2012.

15. Cigarroa RG, Lange RA, Williams RH and Hillis LD: Dosing of contrast material to prevent contrast nephropathy in patients with renal disease. Am J Med 86: 649-652, 1989.

16. Task Force on the management of ST-segment elevation acute myocardial infarction of the European Society of Cardiology (ESC); Steg PG, James SK, Atar D, Badano LP, Blömstrom-Lundqvist C, Borger MA, Di Mario C, Dickstein K, Ducrocq G, Fernandez-Aviles F, et al: ESC Guidelines for the management of acute myocardial infarction in patients presenting with ST-segment elevation. Eur Heart J 33: 2569-619, 2012. 
17. Hamm CW, Bassand JP, Agewall S, Bax J, Boersma E, Bueno H, Caso P, Dudek D, Gielen S, Huber K, et al; ESC Committee for Practice Guidelines: ESC Guidelines for the management of acute coronary syndromes in patients presenting without persistent ST-segment elevation: The Task Force for the management of acute coronary syndromes (ACS) in patients presenting without persistent ST-segment elevation of the European Society of Cardiology (ESC). Eur Heart J 32: 2999-3054, 2011.

18. Liu LS; Writing Group of 2010 Chinese Guidelines for the Management of Hypertension: 2010 Chinese guidelines for the management of hypertension. Zhonghua Xin Xue Guan Bing Za Zhi 39: 579-615, 2011 (In Chinese).

19. Chinese Diabetes Society: 2010 Chinese Guidelines for the Management of Diabetes. Zhonghua Xin Xue Guan Bing Za Zhi 20 S1-S36, 2012 (In Chinese).

20. Ruilope LM, Salvetti A, Jamerson K, Hansson L, Warnold I, Wedel $\mathrm{H}$ and Zanchetti A: Renal function and intensive lowering of blood pressure in hypertensive participants of the hypertension optimal treatment (HOT) study. J Am Soc Nephrol 12: 218-225, 2001.

21. Guo J, Zheng C, Xiao Q, Gong S, Zhao Q, Wang L, He J, Yang W, Shi X, Sun X and Liu J: Impact of anaemia on lung function and exercise capacity in patients with stable severe chronic obstructive pulmonary disease. BMJ Open 5: e008295, 2015.

22. Chinese Society of Cardiology; Editorial Committee of Chinese Journal of Cardiology: Chinese expert consensus on the clinical application of iodinated contrast media in cardiovascular diseases. Zhonghua Xin Xue Guan Bing Za Zhi 41: 94-98, 2013 (In Chinese).

23. Rich MW and Crecelius CA: Incidence, risk factors, and clinical course of acute renal insufficiency after cardiac catheterization in patients 70 years of age or older. A prospective study. Arch Intern Med 150: 1237-1242, 1990.

24. Kini AS, Mitre CA, Kim M, Kamran M, Reich D and Sharma SK: A protocol for prevention of radiographic contrast nephropathy during percutaneous coronary intervention: Effect of selective dopamine receptor agonist fenoldopam. Catheter Cardiovasc Interv 55: 169-173, 2002.

25. Solomon R: The role of osmolality in the incidence of contrast-induced nephropathy: A systematic review of angiographic contrast media in high risk patients. Kidney Int 68: 2256-2263, 2005

26. Barrett BJ and Carlisle EJ: Metaanalysis of the relative nephrotoxicity of high- and low-osmolality iodinated contrast media. Radiology 188: 171-178, 1993.

27. Nozue T, Michishita I, Iwaki T, Mizuguchi I and Miura M: Contrast medium volume to estimated glomerular filtration rate ratio as a predictor of contrast-induced nephropathy developing after elective percutaneous coronary intervention. J Cardiol 54 214-220, 2009

28. Goldenberg I and Matetzky S: Nephropathy induced by contrast media: Pathogenesis, risk factors and preventive strategies. CMAJ 172: 1461-1471, 2005.

29. Chinese society of cardiology, Editorial committee of Chinese journal of cardiology: Chinese expert consensus on the clinical application of iodinated contrast media in cardiovascular diseases. Chinese Journal of Cardiology 41: 94-98, 2013.

30. Alamartine E, Phayphet M, Thibaudin D, Barral FG and Veyret C: Contrast medium-induced acute renal failure and cholesterol embolism after radiological procedures: Incidence, risk factors, and compliance with recommendations. Eur J Intern Med 14: 426-431, 2003

31. Ho KM and Power BM: Benefits and risks of furosemide in acute kidney injury. Anaesthesia 65: 283-293, 2010.
32. Gu GQ, Lu R, Cui W, Liu F, Zhang Y, Yang XH, Chen XF and Jia WM: Low-dose furosemide administered with adequate hydration reduces contrast-induced nephropathy in patients undergoing coronary angiography. Cardiology 125: 69-73, 2013.

33. Toprak $\mathrm{O}$ and Cirit M: Risk factors for contrast-induced nephropathy. Kidney Blood Press Res 29: 84-93, 2006.

34. Toprak O, Cirit M, Yesil M, Byrne DW, Postaci N, Bayata S, Majchrzak KM and Esi E: Metabolic syndrome as a risk factor for contrast-induced nephropathy in non-diabetic elderly patients with renal impairment. Kidney Blood Press Res 29: 2-9, 2006.

35. Liu Y, Tan N, Zhou YL, He PC, Luo JF and Chen JY: The contrast medium volume to estimated glomerular filtration rate ratio as a predictor of contrast-induced nephropathy after primary percutaneous coronary intervention. Int Urol Nephrol 44: 221-229, 2012.

36. Macciò A, Madeddu C, Gramignano G, Mulas C, Tanca L, Cherchi MC, Floris C, Omoto I, Barracca A and Ganz T: The role of inflammation, iron, and nutritional status in cancer-related anemia: Results of a large, prospective, observational study. Haematologica 100: 124-132, 2015.

37. Weiss G and Goodnough LT: Anemia of chronic disease. N Engl J Med 352: 1011-1023, 2005.

38. Waters JS, O'Brien ME and Ashley S: Management of anemia in patients receiving chemotherapy. J Clin Oncol 20: 601-603, 2002.

39. Hsu CY, McCulloch CE and Curhan GC: Epidemiology of anemia associated with chronic renal insufficiency among adults in the United States: Results from the Third National Health and Nutrition Examination Survey. J Am Soc Nephrol 13: 504-510, 2002.

40. Nurko S: Anemia in chronic kidney disease: Causes, diagnosis, treatment. Cleve Clin J Med 73: 289-297, 2006.

41. Astor BC, Coresh J, Heiss G, Pettitt D and Sarnak MJ: Kidney function and anemia as risk factors for coronary heart disease and mortality: The Atherosclerosis Risk in Communities (ARIC) Study. Am Heart J 151: 492-500, 2006.

42. Shah AD, Nicholas O, Timmis AD, Feder G, Abrams KR, Chen R, Hingorani AD and Hemingway H: Threshold haemoglobin levels and the prognosis of stable coronary disease: Two new cohorts and a systematic review and meta-analysis. PLoS Med 8: e1000439, 2011.

43. Carson JL, Noveck H, Berlin JA and Gould SA: Mortality and morbidity in patients with very low postoperative $\mathrm{Hb}$ levels who decline blood transfusion. Transfusion 42: 812-818, 2002.

44. Barbarova I, Klempfner R, Rapoport A, Wasserstrum Y, Goren I, Kats A and Segal G: Avoidance of Blood Transfusion to Patients Suffering From Myocardial Injury and Severe Anemia Is Associated With Increased Long-Term Mortality: A Retrospective Cohort Analysis. Medicine (Baltimore) 29: e1635, 2015.

45. Klein MJ, Carter TI, Smith MC, Wong J and Sugiyama G: Prophylactic hypothermia and neuromuscular blockade to limit myocardial oxygen demand in a critically anemic Jehovah's Witness after emergency surgery. J Surg Case Rep 12: rju135, 2014.

46. McCullough PA and Sandberg KR: Epidemiology of contrast-induced nephropathy. Rev Cardiovasc Med 4 (Suppl 5): S3-S9, 2003.

47. World Health Organization: Haemoglobin concentrations for the diagnosis of anaemia and assessment of severity. In: Vitamin and Mineral Nutrition Information System. WHO, Geneva, pp1-6, 2011. 